\title{
Large-scale computation of the exponentially expanding universe in a simplified Lorentzian type IIB matrix model
}

\author{
Yuta Ito*i \\ Department of Particles and Nuclear Physics, \\ Graduate University for Advanced Studies (SOKENDAI), \\ Tsukuba, Ibaraki 305-0801, Japan \\ E-mail: yito@post.kek.jp

\section{Jun Nishimura} \\ KEK Theory Center, High Energy Accelerator Research Organization, \\ Tsukuba, Ibaraki 305-0801, Japan \\ Department of Particles and Nuclear Physics, \\ Graduate University for Advanced Studies (SOKENDAI), \\ Tsukuba, Ibaraki 305-0801, Japan \\ E-mail: jnishi@post.kek.jp

\section{Asato Tsuchiya} \\ Department of Physics, Shizuoka University, \\ 836 Ohya, Suruga-ku, Shizuoka 422-8529, Japan \\ E-mail: tsuchiya.asato@shizuoka.ac.jp
}

The type IIB matrix model is a conjectured nonperturbative formulation of superstring theory. Recent studies on the Lorentzian version of the model have shown that only three out of nine spatial directions start to expand after some critical time. On the other hand, due to the unbounded action of the Lorentzian model, one has to introduce infrared (IR) cutoffs in order to make the partition function finite. In this work we investigate whether the effects of the IR cutoffs disappear in the infinite volume limit. For that purpose, we study a simplified model with large matrix size up to $N=256$ by Monte Carlo simulation. First we confirm the exponentially expanding behavior of the "universe". Then we generalize the form of the IR cutoffs by one parameter, and find that the results become universal in some region of the parameter. It is suggested that the effects of IR cutoffs disappear in this region, which is confirmed also from the studies of Schwinger-Dyson equations.

The 33rd International Symposium on Lattice Field Theory

14 -18 July 2015

Kobe International Conference Center, Kobe, Japan*

\footnotetext{
* Speaker.

${ }^{\dagger}$ KEK-TH-1879
} 


\section{Introduction}

Superstring theory is a promising candidate of the quantum theory of gravity. Since it requires the space-time to have ten dimensions, it is important to understanding how to get a fourdimensional space-time from the theory. A conventional approach is to compactify six dimensional space using various manifolds. However, it is known that such compactifications give rise to many possible vacua described by various low energy effective theories in $4 \mathrm{~d}$ with different gauge symmetries. Since there is no guiding principle to pick up one particular vacuum as far as one deals with the theory perturbatively, one definitely needs to consider the theory non-perturbatively.

The type IIB matrix model [1] was proposed as a non-perturbative formulation of superstring theory. The important feature of this model is that the $10 \mathrm{~d}$ space-time is described dynamically as the eigenvalue distribution of ten bosonic matrices. Therefore one can investigate what kind of space-time emerges in this model. Until recently, this model has been studied after the Wick rotation since it is known that the partition function of the Euclidean model is finite without any cutoffs. However, the Euclidean model is not suitable for studying the real time dynamics because the time coordinate is treated as purely imaginary. Moreover, a recent study of the Euclidean model using the Gaussian expansion method indicates that the emergent space-time seems to favor three dimensions rather than four dimensions [2].

On the other hand, the Lorentzian version of the type IIB matrix model has been studied by Monte Carlo simulation in ref. [3]. Unlike the Euclidean case, one has to introduce the infrared (IR) cutoffs in both temporal and spatial directions in order to make the partition function finite. Despite such subtleties, one can extract the time evolution of the space-time from configurations generated by Monte Carlo simulation. Remarkably, it turned out that the $\mathrm{SO}(9)$ rotational symmetry of the $9 \mathrm{~d}$ space is spontaneously broken down to $\mathrm{SO}(3)$ at some critical time, after which only three out of nine spatial directions start to expand.

In order to probe further the time evolution of the emergent $3 \mathrm{~d}$ space, one needs to increase the matrix size, which makes the Monte Carlo simulation more and more time-consuming. For this reason, two simplified models have been studied. These models are expected to capture the qualitative behaviors of the expanding space at early times [4, 5] and at late times [6], respectively, in the original model. The results suggested a scenario for the original model that the exponential expanding behavior of $3 \mathrm{~d}$ space continues for a while at early times and it changes into a powerlaw expansion at late times [6]. Thus, in this model, the inflationary universe seems to be naturally realized. Moreover, the suggested power-low expansion at late times seems to be consistent with the $t^{1 / 2}$ behavior, which is reminiscent of the behavior of the Friedman-Robertson-Walker universe in the radiation dominated era.

Let us emphasize here that the interesting properties such as the SSB of $\mathrm{SO}(9)$ and the exponential expansion are obtained after regularizing the model by introducing the IR cutoffs. Therefore it is important to examine whether the effects of the IR cutoffs disappear in the infinite volume limit. This is highly nontrivial since the space-time emerges dynamically in this model. In order to see the trends in the infinite volume limit, we need to use large matrix size, and hence we consider the simplified model for early times in this study. We generalize the form of the IR cutoffs by one parameter, and find that the results become universal in some region of the parameter. It is therefore suggested that the effects of IR cutoffs disappear in this region, which is confirmed also from the 
studies of Schwinger-Dyson equations.

The rest of this article is organized as follows. In section 2 we briefly review the definition of the Lorentzian type IIB matrix model. In section 3 we define the simplified model, which enables us to achieve large matrices in Monte Carlo simulation, and show, in particular, the exponential expansion of space in this model. In section 4 we discuss the expanding behavior of space-time for different choices of the parameter in the IR cutoffs. Section 5 is devoted to a summary and discussions.

\section{Lorentzian version of the type IIB matrix model}

The action of the Lorentzian type IIB matrix model [1] is given by

$$
\begin{aligned}
S_{\mathrm{b}} & =-\frac{1}{4 g^{2}} \operatorname{Tr}\left(\left[A_{\mu}, A_{v}\right]\left[A^{\mu}, A^{v}\right]\right), \\
S_{\mathrm{f}} & =-\frac{1}{2 g^{2}} \operatorname{Tr}\left(\Psi_{\alpha}\left(\mathscr{C} \Gamma^{\mu}\right)_{\alpha \beta}\left[A_{\mu}, \Psi_{\beta}\right]\right),
\end{aligned}
$$

where the bosonic matrices $A_{\mu}(\mu=0, \ldots, 9)$ and the fermionic matrices $\Psi_{\alpha}(\alpha=1, \ldots, 16)$ are $N \times N$, traceless and Hermitian. We have used the $10 \mathrm{~d}$ gamma-matrices after the Weyl projection denoted by $\Gamma^{\mu}$ and the charge conjugation matrix $\mathscr{C}$. The coupling constant $g$ is just a scale parameter in this model since it can be absorbed by rescaling $A_{\mu}$ and $\Psi_{\alpha}$ appropriately. The indices $\mu$ and $v$ are raised and lowered using the Minkowski metric $\eta_{\mu v}=\operatorname{diag}(-1,1, \ldots, 1)$. One can obtain the Euclidean version by making the Wick rotation $A_{0}=i A_{10}$, where $A_{10}$ is supposed to be Hermitian.

The partition function for the Lorentzian version is proposed as [3]

$$
Z=\int d A d \Psi e^{i\left(S_{\mathrm{b}}+S_{\mathrm{f}}\right)},
$$

where the factor " $i$ " in front of the action is motivated from the fact that the string world-sheet metric should also have a Lorentzian signature. Since the Lorentzian version deals with a real time instead of an imaginary time, it is suitable for investigating the real-time dynamics. Note, however, that the bosonic action (2.1) can be written as

$$
S_{\mathrm{b}} \simeq-2 \operatorname{Tr}\left(F_{0 i}\right)^{2}+\operatorname{Tr}\left(F_{i j}\right)^{2},
$$

where we have introduced the Hermitian matrices $F_{\mu \nu}=i\left[A_{\mu}, A_{v}\right]$. Therefore $S_{\mathrm{b}}$ is no longer positive semi-definite, and it is not bounded from below because the two terms in the action have opposite signs.

In order to make the partition function (2.3) finite, one needs to regularize it by introducing IR cutoffs in both temporal and spatial directions, for instance, as

$$
\begin{aligned}
& \frac{1}{N} \operatorname{Tr}\left[\left(A_{0}^{2}\right)^{p}\right] \leq \kappa^{p}, \\
& \frac{1}{N} \operatorname{Tr}\left[\left(A_{i}^{2}\right)^{p}\right] \leq 1 .
\end{aligned}
$$

While we have used $p=1$ for simplicity in the previous work [3, 4, 5], it is important to examine whether the obtained results depend on the choice of $p$ in the infinite volume limit. 
The partition function (2.3) can be rewritten into a form which allows direct Monte Carlo studies in the following way. (See appendix A of ref. [5] for details.) First, by integrating out the fermionic matrices in the partition function $(2.3$, we obtain the Pfaffian

$$
\int d \Psi e^{i S_{\mathrm{f}}}=\operatorname{Pf} \mathscr{M}(A)
$$

which is real unlike in the Euclidean case. Since $S_{\mathrm{b}}$ becomes $r^{4} S_{\mathrm{b}}$ under rescaling $A_{\mu} \mapsto r A_{\mu}$, we can think of integrating out the scale factor of $A_{\mu}$ first. Then the phase factor $e^{i S_{\mathrm{b}}}$ can be converted into a delta function $\delta\left(S_{\mathrm{b}}\right)$ for sufficiently large matrix size, and we arrive at

$$
Z=\int d A \operatorname{Pf} \mathscr{M}(A) \delta\left(S_{\mathrm{b}}\right) \delta\left(\frac{1}{N} \operatorname{Tr}\left[\left(A_{i}^{2}\right)^{p}\right]-1\right) \theta\left(\kappa^{p}-\frac{1}{N} \operatorname{Tr}\left[\left(A_{0}^{2}\right)^{p}\right]\right) .
$$

Here the delta function and Heaviside step function $\theta(x)$ represent the IR cutoffs (2.4) and 2.5).

In order to extract the time evolution from configurations generated by simulating [2.7), we diagonalize the temporal matrix $A_{0}$ as

$$
A_{0}=\operatorname{diag}\left(\alpha_{1}, \ldots, \alpha_{N}\right), \quad \text { where } \alpha_{1}<\cdots<\alpha_{N},
$$

using the $\mathrm{SU}(N)$ symmetry of the model. It turns out that the spatial matrices $A_{i}$ have a banddiagonal structure in this basis. In other words, there exists some integer $n$ such that the magnitude of elements of the spatial matrices $\left(A_{i}\right)_{a b}$ for $|a-b|>n$ are much smaller than those for $|a-b|<n$. Based on this observation, we may naturally consider $n \times n$ matrices

$$
\left(\bar{A}_{i}\right)_{I J}(t)=\left(A_{i}\right)_{v+I, v+J}
$$

where $I, J=1, \ldots, n$ and $v=0,1, \ldots, N-n$, and $t$ is the time defined by

$$
t=\frac{1}{n} \sum_{I=1}^{n} \alpha_{v+I}
$$

corresponding to the $n \times n$ matrices $\bar{A}_{i}$. We regard $\bar{A}_{i}(t)$ as the state of the universe at time $t$. Using (2.9), for example, we can define the extent of the space at time $t$ as

$$
R^{2}(t)=\left\langle\frac{1}{n} \operatorname{tr} \sum_{i}\left(\bar{A}_{i}(t)\right)^{2}\right\rangle,
$$

where the symbol tr represents a trace over the $n \times n$ block matrix. We can also define the "moment of inertia tensor" as

$$
T_{i j}(t)=\frac{1}{n} \operatorname{tr}\left(\bar{A}_{i}(t) \bar{A}_{j}(t)\right),
$$

whose nine eigenvalues $\lambda_{i}(t)$ represent the spatial extent in each of the nine directions at time $t$.

\section{The simplified model}

The most time-consuming part of the Monte Carlo simulation comes from the Pfaffian. Let us note here that the fermionic action (2.2) can be decomposed into two terms as

$$
S_{\mathrm{f}} \propto \operatorname{Tr}\left(\bar{\Psi}_{\alpha}\left(\Gamma^{0}\right)_{\alpha \beta}\left[A_{0}, \Psi_{\beta}\right]\right)+\operatorname{Tr}\left(\bar{\Psi}_{\alpha}\left(\Gamma^{i}\right)_{\alpha \beta}\left[A_{i}, \Psi_{\beta}\right]\right) .
$$



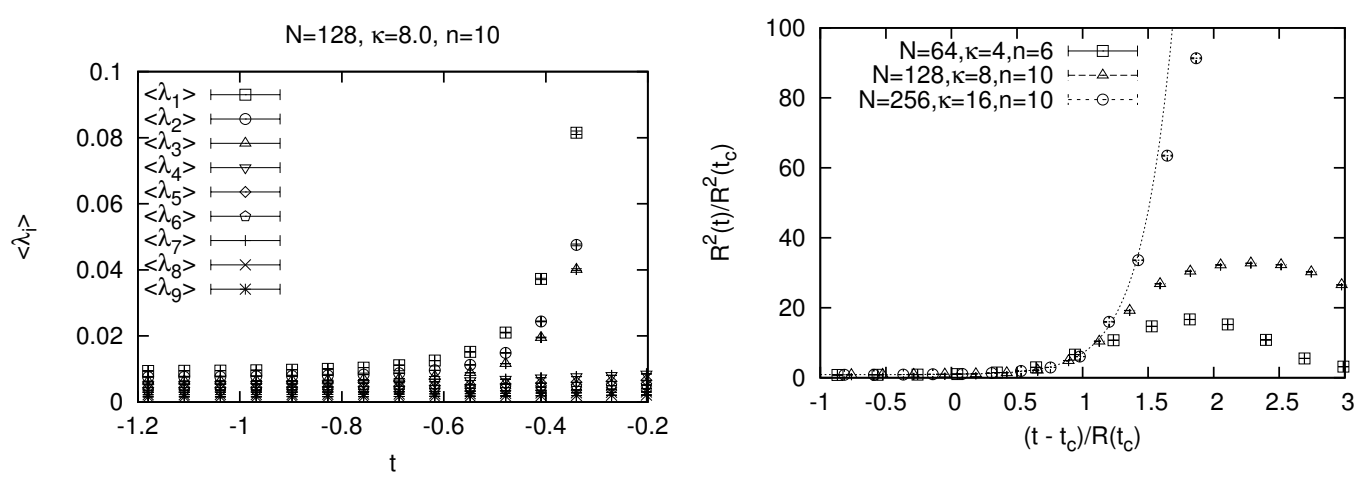

Figure 1: (Left) The nine eigenvalues of $T_{i j}(t)$ are plotted against time $t$ for the simplified model with $N=$ $128, \kappa=8.0, n=10$ using the IR cutoffs (2.4) and (2.5) with $p=1$. (Right) The extent of the space $R^{2}(t)$ normalized by $R^{2}\left(t_{\mathrm{c}}\right)$ is plotted against $x=\left(t-t_{\mathrm{c}}\right) / R\left(t_{\mathrm{c}}\right)$ for the simplified model with $N=64,128$ and 256 using the IR cutoffs (2.4) and (2.5) with $p=1$. The dashed line is a fit to $R^{2}(t) / R^{2}\left(t_{\mathrm{c}}\right)=a+(1-a) \exp (b x)$ with $N=256$ for $0 \leq x \leq 1.3$, which gives $a=0.89(3)$ and $b=4.0(3)$.

At early times, the second term is less important than the first one since the expansion of the universe has not proceeded much. Therefore we may omit the second term as a simplification. Then the Pfaffian simplifies to

$$
\operatorname{Pf} \mathscr{M}(A) \simeq \Delta^{16}(\alpha)
$$

where $\Delta(\alpha) \equiv \prod_{i>j}\left(\alpha_{i}-\alpha_{j}\right)$ is the van der Monde determinant. The partition function of the simplified model is therefore given as

$$
Z=\int d A_{i} \prod_{m} d \alpha_{m} \Delta^{18}(\alpha) \delta\left(S_{\mathrm{b}}\right) \delta\left(\frac{1}{N} \operatorname{Tr}\left[\left(A_{i}^{2}\right)^{p}\right]-1\right) \theta\left(\kappa^{p}-\frac{1}{N} \operatorname{Tr}\left[\left(A_{0}^{2}\right)^{p}\right]\right)
$$

where the extra factor $\Delta^{2}(\alpha)$ comes from the Fadeev-Popov procedure for the gauge fixing.

This simplification allows us to study large matrix size. In Fig. 1(Left), we plot the expectation values $\left\langle\lambda_{i}(t)\right\rangle$ of the nine eigenvalues of $T_{i j}(t)$ for the simplified model with $N=128$ using the IR cutoffs (2.4) and (2.5) with $p=1$. We observe a gap between $\left\langle\lambda_{3}(t)\right\rangle$ and $\left\langle\lambda_{4}(t)\right\rangle$, which signals the spontaneous symmetry breaking of $\mathrm{SO}(9)$ to $\mathrm{SO}(3)$. We define the critical time $t_{\mathrm{c}}$ as the time at which the gap between $\left\langle\lambda_{3}(t)\right\rangle$ and $\left\langle\lambda_{4}(t)\right\rangle$ starts to develop. For example, we obtain $t_{\mathrm{c}}=-0.63108$ (7) for $N=128$ from Fig. 1(Left). Applying the same procedure to other $N$, we find reasonable large- $N$ scaling behaviors for the extent of space $R(t)$. Considering the ambiguities in defining the critical time at finite $N$, we modify the value of $t_{\mathrm{c}}$ slightly from the one determined by the above procedure in such a way that the scaling behavior is optimized. In Fig 1(Right), we plot the extent of the space $R(t)$ obtained in this way. We find that the behavior of $R^{2}(t)$ at $t>t_{\mathrm{c}}$ can be fitted to an exponential function. ${ }^{1}$ It is suggested that the exponential expansion occurs also in the original Lorentzian type IIB matrix model at early times. The results obtained up to $N=24$ for the original model [4] are consistent with this speculation.

\footnotetext{
${ }^{1}$ The exponential expansion has been observed also in the $6 \mathrm{~d}$ version of the simplified model with $N \leq 64$ [5].
} 


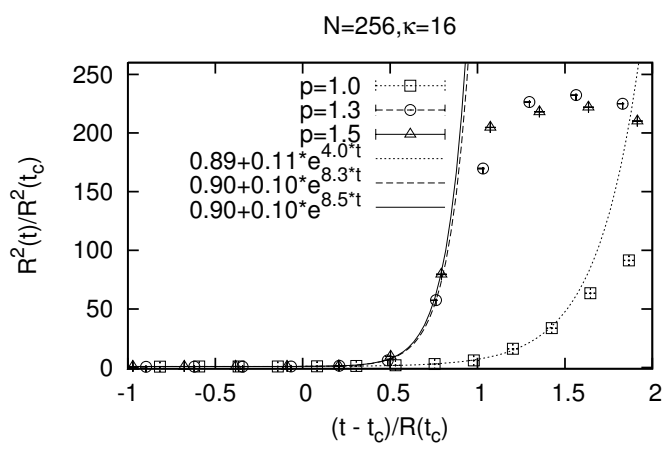

Figure 2: The extent of the space $R^{2}(t)$ normalized by $R^{2}\left(t_{\mathrm{c}}\right)$ is plotted against $x=\left(t-t_{\mathrm{c}}\right) / R\left(t_{\mathrm{c}}\right)$ with $N=256$ and $\kappa=16$ for $p=1.0,1.3$ and 1.5. We have used the block size $n=10$ for $p=1.0$ and $n=6$ for $p=1.3$ and 1.5. The lines are fits to $R^{2}(t) / R^{2}\left(t_{\mathrm{c}}\right)=a+(1-a) \exp (b x)$.

\section{Dependence on the IR cutoffs}

In the previous section, we have shown the results obtained for the simplified model using $p=1$ in the IR cutoffs 2.4 and 2.5. In this section we discuss how the results depend on the parameter $p$ in the IR cutoffs.

In Fig. 2 we plot $R^{2}(t)$ against $t$ for various values of $p$. We find that the results for $p=1.3$ and 1.5 seem to be almost the same except for the region around the peak. On the other hand, the result for $p=1.0$ deviates from the others in the whole $t>t_{\mathrm{c}}$ region. These observations suggest that the effects of the IR cutoffs disappear for sufficiently large $p$ but not for $p=1.0$.

Let us note that, as the power $p$ in (2.4) and (2.5) is increased, the IR cutoffs tend to affect only large eigenvalues of $\left(A_{0}\right)^{2}$ and $\left(A_{i}\right)^{2}$, respectively. This naturally explains why we observe a universal behavior at sufficiently large $p$. From this point of view, we may conclude that the results for $p=1.3$ and 1.5 are affected by the IR cutoffs only around the peak, whereas the results for $p=1.0$ are affected by the IR cutoffs in the whole time region.

We can fit the results for $p=1.3$ and 1.5 by an exponential function as shown in Fig. 2. Thus we find that the result for $p=1.0$ is qualitatively the same as the ones for $p=1.3$ and 1.5.

\section{Summary and discussions}

In this work, we have studied the issues concerning the IR cutoffs introduced to regularize the Lorentzian type IIB matrix model. While the model exhibits interesting properties such as the spontaneous symmetry breaking of $\mathrm{SO}(9)$, it is important to examine whether the effects of the IR cutoffs disappear in the infinite volume limit.

In order to address this issue, we studied the simplified model for early times, which is obtained from the original model by omitting the term proportional to $A_{i}$ in the fermionic action. It turned out that this simplified model also has the properties such as the $\mathrm{SSB}$ of $\mathrm{SO}(9)$ down to $\mathrm{SO}(3)$. We have also confirmed the exponential expansion of space using large matrix size up to $N=256$.

In order to investigate the effects of the IR cutoffs in such results, we have introduced the parameter $p$ in (2.4) and (2.5), where $p=1$ corresponds to the IR cutoffs used so far. The qualitative 
behavior of $R(t)$ is the same for all the values of $p$ we studied. Quantitatively, we find that the results for $p=1.3$ and 1.5 seem to be almost the same except for the region near the peak, whereas the result for $p=1$ is different from the others. This can be understood from the fact that the IR cutoffs tend to affect only the large eigenvalues of $\left(A_{0}\right)^{2}$ and $\left(A_{i}\right)^{2}$ as $p$ is increased. Thus our results suggest that the IR cutoff effects disappear in the scaling region and the results become universal for sufficiently large $p$ but not for $p=1.0$.

As a more direct approach to probe the effects of the IR cutoffs, we have also studied SchwingerDyson equations. According to this analysis, we indeed find that the term arising from the IR cutoffs becomes smaller as $N$ is increased when $p>1$. This also suggests that the effects of the IR cutoffs disappear in the infinite volume limit for sufficiently large $p$.

Considering that the argument on the disappearance of the cutoff effects does not depend on the details of the model, we may naturally expect that the cutoff effects also disappear with sufficiently large $p$ for the original model. However, the critical value of $p$ beyond which the cutoff effects disappear in the infinite volume limit may depend on the model. It is therefore important to determine the critical $p$ by simulating the original model.

\section{Acknowledgments}

This research used computational resources of the K computer of the HPCI system provided by the AICS through the HPCI System Research Project (Project ID : hp130063). The supercomputer FX10 at University of Tokyo has been used in developing our code for parallel computing. The work of Y.I. is supported by Grant-in Aid for JSPS fellows. The work of J. N. and A. T. was supported in part by Grant-in-Aid for Scientific Research (No. 23244057 for J. N. and No. 24540264, 23244057, 15K05046 for A. T.) from Japan Society for the Promotion of Science.

\section{References}

[1] N. Ishibashi, H. Kawai, Y. Kitazawa, and A. Tsuchiya, A large-N reduced model as superstring, Nucl. Phys. B, bf 498, 467 (1997) [arXiv: hep-th/9612115].

[2] J. Nishimura, T. Okubo and F. Sugino, Systematic study of the SO(10) symmetry breaking vacua in the matrix model for type IIB superstrings, JHEP 1110 (2011) 135 [arXiv: 1108.1293 [ hep-th] ].

[3] S. W. Kim, J. Nishimura and A. Tsuchiya, Expanding (3+1)-dimensional universe from a Lorentzian matrix model for superstring theory in (9+1)-dimensions, Phys. Rev. Lett. 108 (2012) 011601 [arXiv:1108.1540 [hep-th]].

[4] Y. Ito, S. W. Kim, J. Nishimura and A. Tsuchiya, Monte Carlo studies on the expanding behavior of the early universe in the Lorentzian type IIB matrix model, PoS LATTICE 2013 (2014) 341 [arXiv:1311.5579 [hep-lat]].

[5] Y. Ito, S. W. Kim, Y. Koizuka, J. Nishimura and A. Tsuchiya, A renormalization group method for studying the early universe in the Lorentzian IIB matrix model, PTEP 2014 (2014) 8, $083 \mathrm{~B} 01$ [arXiv:1312.5415 [hep-th]].

[6] Y. Ito, J. Nishimura and A. Tsuchiya, Power-law expansion of the Universe from the bosonic Lorentzian type IIB matrix model, JHEP 1511 (2015) 070 [arXiv: 1506.04795 [hep-th] ]. 